\title{
Fallschwere und SwissDRG
}

Eineinhalb Jahre nach Einführung der neuen Spitalfinanzierung, zeigt die Ärzteschaft vermehrtes Interesse an Informationen zu SwissDRG. Erfährt sie doch im Spitalalltag, dass schwerkranke Patienten hohe Kosten verursachen, aber nicht immer ausreichend vergütet werden und somit die finanzielle Lage eines Spitals erheblich beeinflussen können.

\section{Bettina Holzer}

Ressort Tarife und Gesundheitsökonomie Spitalärzte
Im SwissDRG-System gibt es verschiedene Möglichkeiten, Begleiterkrankungen, Komplikationen und komplexe Behandlungssituationen abzubilden, die während des stationären Aufenthaltes auftreten. Der behandelnde Arzt benötigt neben dem ärztlichen Fachwissen auch die Kenntnis, wie sich solche Fälle im neuen Tarifsystem abbilden lassen. Die Grundlageninformationen des Fallpauschalensystems wurden im Artikel «SwissDRG - das Wichtigste in Kürze» erläutert. [1]

\section{Komplikation und Komorbidität (CC) - der Schweregrad einer Nebendiagnose}

Komplikationen und/oder Komorbiditäten (CC) [2] sind in der Regel relevante Nebendiagnosen, die eine Behandlung von Krankheiten erschweren und in Folge einen höheren Ressourcenverbrauch nach sich

Tabelle 1

Schweregrad einer Komplikation oder Komorbidität.

CCL Schweregrad

0 keine Komplikation oder Komorbidität

1 leichte Komplikation oder Komorbidität

2 mässig schwere Komplikation oder Komorbidität

3 schwere Komplikation oder Komorbidität

4 äusserst schwere Komplikation oder Komorbidität ziehen. Für alle Nebendiagnosen sind Schweregradstufen (CCL) [3] vorgegeben, deren Wert zwischen 0 und 4 für operative und neonatologische Behandlungsepisoden sowie zwischen 0 und 3 für medizinische Behandlungsepisoden variieren kann (Tab. 1). Der Grouper ermittelt in einem komplexen Verfahren für jede Diagnose in einem Datensatz den CCLWert [4]. Dieser Wert hängt auch von der Hauptdiagnose ab, so dass mit der Hauptdiagnose verwandte Nebendiagnosen ihren Wert verlieren.

Nur Nebendiagnosen mit einem CCL-Wert $>0$ sind von Bedeutung. In der SwissDRG Version 3.0 sind ca. $25 \%$ aller Diagnosecodes mit einem CCL-Wert $>0$ gekennzeichnet. [5] Eine unspezifische Nebendiagnose, in der Regel in der ICD 10 benannt mit der Endziffer 9 und im Text als «nicht näher bezeichnet», hat häufig einen geringeren CCL-Wert als eine spezifische Diagnose oder keinen CCL-Wert (Wert $=0$ ).

Beispielsweise hat die unspezifische nicht näher bezeichnete chronisch obstruktive Lungenkrankheit (ICD 10 J44.90) im Vergleich zu der spezifischen chronisch obstruktiven Lungenerkrankung mit akuter Infektion der unteren Atemwege (ICD 10 J44.00) keine CC-Relevanz (Tab. 2) [6].

Die Tabelle zum Nachschlagen der CCL-Werte der Diagnosen, genannt CCL-Matrix, kann sich als Ergebnis der Überarbeitung und Neukalkulation von Systemversion zu Systemversion ändern. Beispielsweise können Nebendiagnosen, die im Vorjahr eine

\section{Tabelle 2}

Abhängigkeit des CCL-Wertes von der Diagnose.

\begin{tabular}{|c|c|c|c|c|}
\hline ICD10 & $\begin{array}{l}\text { Beschreibung } \\
\text { Diagnose }\end{array}$ & $\begin{array}{l}\text { Detailbeschreibung } \\
\text { Diagnose }\end{array}$ & $\begin{array}{l}\text { CCL Operative } \\
\text { Partition einer } \\
\text { DRG }\end{array}$ & $\begin{array}{l}\text { CCL Medizini- } \\
\text { sche Partition } \\
\text { einer DRG }\end{array}$ \\
\hline J44.00, J44.01, J44.02 & $\begin{array}{l}\text { Chronisch obstruktive } \\
\text { Lungenkrankheit mit akuter } \\
\text { Infektion der unteren } \\
\text { Atemwege }\end{array}$ & $\begin{array}{l}\text { FEV1 (forciertes exspiratorisches } \\
\text { Volumen) je nach Diagnose }<35 \% \\
\text { bis }<70 \%\end{array}$ & 2,3 & 2,3 \\
\hline J44.03 & dito & FEV1 $\geq 70 \%$ & 2 & 1,2 \\
\hline J44.09 & dito & FEV1 nicht näher bezeichnet & 2 & 1,2 \\
\hline $\begin{array}{l}\mathrm{J} 44.90, \mathrm{~J} 44.91, \mathrm{~J} 44.92 \\
\mathrm{~J} 44.93, \mathrm{~J} \\
\end{array}$ & $\begin{array}{l}\text { chronische obstruktive } \\
\text { Lungenkrankheit, nicht näher } \\
\text { bezeichnet }\end{array}$ & unabhängig von der FEV1 & 0 & 0 \\
\hline
\end{tabular}

Korrespondenz: Dr. med. Bettina Holzer FMH

Froburgstrasse 15 CH-4600 Olten Tel. 0313591111 Fax 0313591112 tarife.spital[at]fmh.ch 


\section{Aktuelle}

Forumthemen

Diskutieren Sie mit! Im Forum präsentieren wir regelmässig brisante Themen aus Politik, Ökonomie und Wissenschaft, die das Schweizer Gesundheitswesen betreffen. Bringen Sie Ihre Meinung ein oder kommentieren Sie die Äusserungen Ihrer Kolleginnen und Kollegen. Das Forum finden Sie unter: www.saez.ch/forum/
Bedeutung hatten, im folgenden Jahr abgewertet werden und/oder umgekehrt neue Nebendiagnosen aufgewertet werden. So wurde in der SwissDRG-Version 3.0 der CCL-Wert des Kodes «Blutung und Hämatom als Komplikation eines Eingriffes, andernorts nicht klassifiziert» (ICD10 T81.0) für die operativen und medizinischen Fälle mit $\mathrm{CCL}=1$ deutlich abgewertet. In der SwissDRG-Version 2.0 hatte dieser noch die CCL-Werte 2,3,4 in der operativen bzw. 2,3 in der medizinischen Partition einer DRG.

\section{Patientenbezogener Schweregrad (PCCL) - der Schweregrad des Behandlungsfalles}

Kumuliert man die CCL-Werte der Nebendiagnosen eines Patienten, so erhält man den PCCL-Wert des Behandlungsfalles [7]. Man unterscheidet dabei Schweregradstufen von 0 bis 4 (Tab. 3). Die Ermittlung des PCCL-Wertes erfolgt in einem komplexen Verfahren. Dabei wird darauf geachtet, dass ähnliche Umstände nicht mehrfach gewertet werden. So führen z.B. 5 «leichte» Nebendiagnosen mit je einem CCL-Wert von $1 \mathrm{zu}$ einem PCCL-Gesamtschweregrad von 3 mit schweren Komplikationen oder Komorbiditäten, genauso wie die Kombination einer «leichten» $(\mathrm{CCL}=1)$ und einer «mässig schweren» Nebendiagnose $(\mathrm{CCL}=2)$ [8].

Ein gesunder Patient ohne Begleiterkrankungen, der sich wegen eines Gallenblasensteines mit akuter Cholezystitis (ICD 10 K80.00) einer komplikationslosen laparoskopischen Gallenblasenentfernung unterzieht, hat im SwissDRG-System 3.0 einen PCCLWert von 0 . Hat er zusätzlich jedoch eine nicht nä-

Tabelle 3
Patientenbezogener Gesamtschweregrad.
\begin{tabular}{ll} 
PCCL & Schweregrad \\
\hline 0 & keine Komplikation oder Komorbidität \\
\hline 1 & leichte Komplikation oder Komorbidität \\
\hline 2 & mässig schwere Komplikation oder Komorbidität \\
\hline 3 & schwere Komplikation oder Komorbidität \\
\hline 4 & äusserst schwere Komplikation oder Komorbidität
\end{tabular}

Tabelle 4

ADRG 109 - DRG-Split anhand von Schweregraden [9].

\begin{tabular}{ll} 
ADRG 109 & $\begin{array}{l}\text { Wirbelkörperfusion } \\
\text { Prozedur in Tabelle 109-1 }\end{array}$ \\
\hline DRG 109A & $\begin{array}{l}\text { Wirbelkörperfusion mit äusserst schweren CC } \\
\text { PCCL }>3\end{array}$ \\
\hline DRG 109B & $\begin{array}{l}\text { Wirbelkörperfusion mit schweren CC } \\
\text { PCCL }>2\end{array}$ \\
DRG 109C & Wirbelkörperfusion ohne äusserst schwere oder schwere CC
\end{tabular}

Prozedurentabelle 109-1

03.53.11 $\leftrightarrow$ geschlossene Reposition einer Wirbelfraktur mit externer Fixierung

03.53.20 $\leftrightarrow$ offene Reposition einer Wirbelfraktur

78.49.20↔Wirbelkörperersatz (z.B. bei Kyphose), nicht näher bezeichnet usw. her bezeichnete chronische Bronchitis (ICD $10 \mathrm{~J} 42$ ), so hat er einen PCCL-Wert von 1. Liegt ausserdem noch ein Diabetes mellitus mit Nierenkomplikation, nicht entgleist (ICD 10 E11.20) vor, so erhöht sich sein PCCL-Wert auf 3, und tritt als Komplikation zusätzlich eine Sepsis mit Staphylococcus aureus (ICD 10 A41.0) auf, so resultiert ein PCCL-Wert von 4.

\section{Komorbiditäten ermöglichen kosten- trennende DRG-Splits}

Der PCCL-Wert wird in vielen Basis-DRGs zur Differenzierung der Schweregrade einer DRG verwendet (Tab. 4).

Die SwissDRG-Version 3.0 umfasst insgesamt 988 DRGs, darunter finden sich 24 DRGs «mit schweren CC», d.h. mit einem PCCL >2, sowie 141 DRGs «mit äusserst schweren CC», d.h. mit einem PCCL $>3$.

\section{Weitere Abbildungsmöglichkeiten für spezifische und hochkomplexe Behandlungs- situationen}

Neben dem CCL-System kann das DRG-System erschwerende Begleitumstände bei bestimmten Behandlungssituationen auch durch komplizierende und komplexe Diagnosen abbilden. In den Basis-DRGs zugeordneten Tabellen sind diese Konstellationen ersichtlich. 30 DRGs mit einer oder mehreren komplizierenden Diagnosen sowie 39 DRGs mit komplexen Diagnosen werden in der SwissDRG-Version 3.0 aufgeführt wie beispielsweise die DRG E66A «schweres Thoraxtrauma mit komplizierender Diagnose». Als Splitkriterium von DRG E66B «schweres Thoraxtrauma ohne komplizierende Diagnose» zur höher bewerteten DRG E66A «schweres Thoraxtrauma mit komplizierender Diagnose» kommt zum Beispiel eine gleichzeitig zur Hauptdiagnose «Rippenserienfraktur» vorkommende komplizierende Nebendiagnose «Brustwirbelfraktur» zum Tragen [10].

Spezifische und komplexe Behandlungen werden im SwissDRG-System auch über die sogenannten Funktionen abgebildet. Diese Funktionen sind Zuordnungslogiken und Kodes, die an unterschiedlichen Stellen in den DRG Algorithmus eingreifen sowie als Kostentrenner für spezifische und hochkomplexe Behandlungssituationen fungieren und so auf unterschiedliche Weise einen Mehraufwand abbilden (Tab. 5). In der vorliegenden SwissDRG-Version 3.0 sind 18 Funktionen definiert, 2 davon sind spezifisch für Neugeborene, 16 sind sogenannte globale Funktionen. Details, in welchen MDC [11] und DRGs die einzelnen Funktionen ihre Verwendung finden, sind im SwissDRG-Definitionshandbuch Version 3.0 in Band 3, 4 und 5 dargestellt [12].

Somit wird ersichtlich, dass der Aufwand für Patienten, die auf einer Intensivstation behandelt werden oder eine geriatrische frührehabilitative Komplexbehandlung erhalten, grösser und damit teurer ist als der Aufwand für Patienten, bei denen 
keine erschwerenden Begleitumstände bei bestimmten Behandlungssituationen vorliegen.

Es ist daher unerlässlich, unterdeckte Fälle des Spitals auf Einzelfallebene hinsichtlich der Qualität der medizinischer Dokumentation und der Kodierung zu analysieren. Nicht nur, ob alle Nebendiagnosen und Prozeduren korrekt erfasst wurden, sondern auch, ob eine oder mehrere Diagnosen als mögliches DRG-Splitkriterium durch eine komplizierende oder komplexe Diagnose identifiziert werden könnten. Auch eine Prozedur, die nicht aufwandsgerecht in einer zugeordneten DRG plaziert ist und einer anderen DRG zur präziseren Fallabbildung in der zugehörigen Definitionstabelle zugeordnet werden sollte, kann sich als Kostentrenner erweisen. So generieren sich neue DRG-Anträge, die datengestützt von der SwissDRG AG auf der Grundlage der Falldaten der Spitäler für die jährliche Weiterentwicklung des SwissDRG Systems geprüft werden. Ziel bleibt die leistungsgerechte Abbildung medizinischer Leistungen.

\section{Neue Allianzen im Spital - Arzt, Medizin- controlling und Kodierung}

Nur mit einer präzisen Leistungs- und Kostenerfassung auf den Einzelfall kann eine leistungsgerechte Vergütung erzielt werden. Die Anforderungen an die Komplexität und den Detaillierungsgrad der medizinischen Dokumentation sowie der medizinischen Kodierung sind gestiegen. Daher ist eine etablierte Informations- und Dokumentationsstrategie im Spital notwendig. Das Medizincontrolling stellt das Bindeglied zwischen dem administrativen und dem klinischen Bereich des Spitals dar. Die Analyse der durchschnittlichen Schwere der Fälle in einer DRG anhand der PCCL-Verteilung im Vergleich zum Benchmark kann Hinweise auf infrastrukturelle oder kodiertechnische Besonderheiten geben. Besonders Langliegerfälle, Patienten mit Aufenthalt auf einer Intensiv-, Intermediate-Care- oder Schlaganfallstation sowie Patienten mit hohen Material- und Medi-

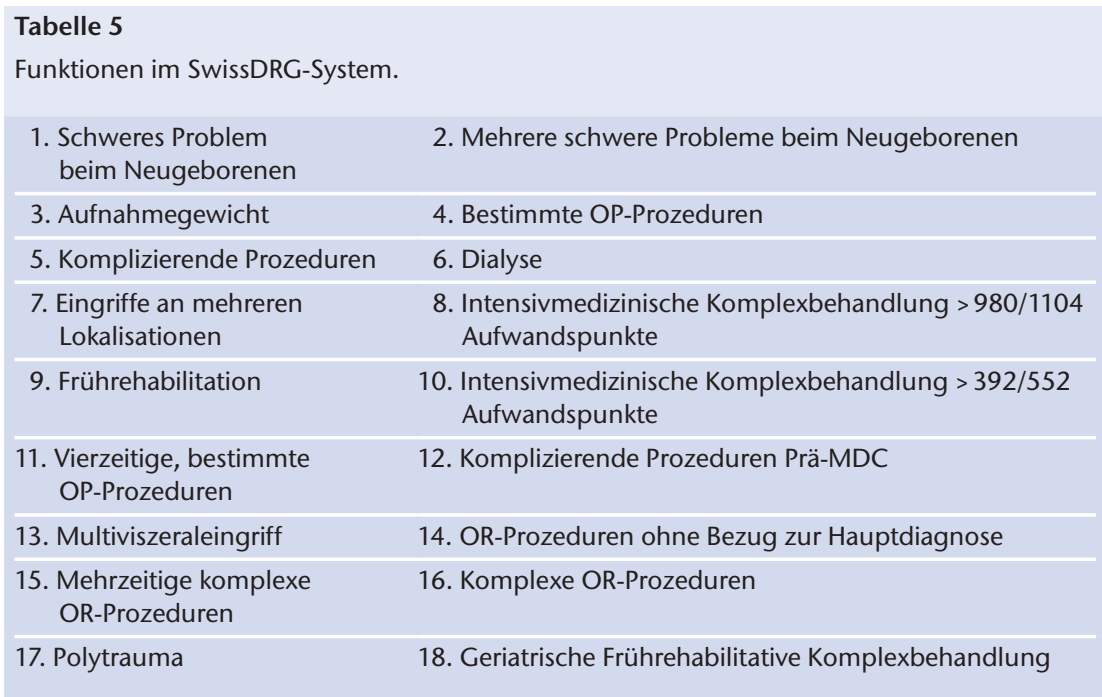

kamentenkosten benötigen eine profunde Analyse. Die regelmässige Standortbestimmung von Dokumentations- und Kodierqualität sowie die frühzeitige Identifikation von möglichen Erlösrisiken im fachabteilungsbezogenen Leistungsspektrum, führt im regelmässigen Austausch zwischen den behandelnden Ärzten, Medizincontrolling und Kodierung zu einer aufwandsgerechteren Leistungsabbildung, aber auch zur Identifizierung von Schwachstellen im DRG-System. Zur Weiterentwicklung eines hochwertigen SwissDRG-Systems bedarf es daher qualifizierter DRG-Anträge mit Darlegung von validen Kostenund Leistungsdaten. Gerne unterstützen Sie die DRG-und Kodierexperten der FMH auch dieses Jahr wieder bei Ihren DRG-Anträgen. Gestalten Sie über Ihre Fachgesellschaft oder Ihren Dachverband das Tarifsystem mit, und reichen Sie bis 5. Juli 2013 Ihre DRG-Anträge bei der FMH ein! [13]]

\section{Literatur}

1 Holzer B. 2012: SwissDRG - das Wichtigste in Kürze. Schweiz Ärztezeitung 93:29/30 1079. Oder www.fmh.ch $\rightarrow$ stationäre Tarife $\rightarrow$ Publikationen $\rightarrow 2012 \rightarrow$ SwissDRG - das Wichtigste in Kürze

2 CC: Complication and Comorbidity, Komplikation und Komorbidität.

3 CCL: Complication and Comorbidity Level, Komplikations- und Komorbiditäts-Schweregrad.

$4 \quad$ www.swissdrg.org $\rightarrow$ SwissDRG System 3.0 $\rightarrow$ Definitionshandbuch SwissDRG 3.0 $\rightarrow$ Katalogversion $(2011 / 2014) \rightarrow$ Bd. $5 \rightarrow$ Anhang C $\rightarrow$ CCs, CC-Ausschlüsse, CCL- und PCCL-Berechnung.

5 www.swissdrg.org $\rightarrow$ SwissDRG System 3.0 $\rightarrow$ Definitionshandbuch SwissDRG 3.0 $\rightarrow$ Katalogversion $(2011 / 2014) \rightarrow$ Bd. $5 \rightarrow$ Anhang C $\rightarrow$ CCs, CC-Ausschlüsse, CCL- und PCCL-Berechnung $\rightarrow$ Einleitung: 3468 von rund 13300 ICD-10-Diagnosecodes bei Erwachsenen, 3286 von rund 13300 ICD-10-Diagnosecodes bei Neugeborenen.

6 www.swissdrg.org $\rightarrow$ SwissDRG System 3.0 $\rightarrow$ Definitionshandbuch SwissDRG 3 .0 $\rightarrow$ Katalogversion $(2011 / 2014) \rightarrow$ Bd. $5 \rightarrow$ Anhang C $\rightarrow$ CCs, CC-Ausschlüsse, CCL- und PCCL-Berechnung.

7 PCCL: Patient Complication and Comorbidity Level, patientenbezogener Gesamtschweregrad.

8 www.swissdrg.org $\rightarrow$ SwissDRG System 3.0 $\rightarrow$ Definitionshandbuch $3.0 \rightarrow$ Katalogversion $(2011 / 2014) \rightarrow$ Band $5 \rightarrow$ Anhang C $\rightarrow$ CCs, CC-Ausschlüsse, CCL- und PCCL-Berechnung sowie Begleitdokumente.

9 www.swissdrg.org $\rightarrow$ SwissDRG-System 3.0 $\rightarrow$ Definitionshandbuch $3.0 \rightarrow$ Katalogversion $(2011 / 2014) \rightarrow$ Band $2 \rightarrow$ MDC $08 \rightarrow$ ADRG I09.

10 www.swissdrg.org $\rightarrow$ SwissDRG System 3.0 $\rightarrow$ Definitionshandbuch $3.0 \rightarrow$ Katalogversion $(2011 / 2014) \rightarrow$ Band $1 \rightarrow$ MDC 04.

11 MDC: Major Diagnostic Category, Hauptdiagnosegruppen im DRG System.

12 www.swissdrg.org $\rightarrow$ SwissDRG System 3.0 $\rightarrow$ Definitionshandbuch $3.0 \rightarrow$ Katalogversion $(2011 / 2014) \rightarrow$ Band $3 \rightarrow$ MDC15 $\rightarrow$ Neugeborene $\rightarrow$ Probleme, und Band $4 \rightarrow$ globale Funktionen, und Band $5 \rightarrow$ Anhang B $\rightarrow$ Funktionsübersicht.

13 www.fmh.ch $\rightarrow$ stationäre Tarife $\rightarrow$ SwissDRG $\rightarrow$ Antragsverfahren. 